\title{
Identification of rare and common variants in BNIP3L: a schizophrenia susceptibility gene
}

\author{
Juan Zhou ${ }^{1+} \mathbb{D}$, Chuanchuan Ma ${ }^{1 \dagger}$, Ke Wang ${ }^{1}$, Xiuli Li', Xuemin Jiann ${ }^{1}$, Han Zhang ${ }^{1}$, Jianmin Yuan², Jiajun Yin², \\ Jianhua Chen ${ }^{3,4^{*}}$ and Yongyong Shi ${ }^{*}$
}

\begin{abstract}
Background: Schizophrenia is a chronic and severe mental disorder, and it has been predicted to be highly polygenic. Common SNPs located in or near BNIP3L were found to be genome-wide significantly associated with schizophrenia in recent genome-wide association studies. The purpose of our study is to investigate potential causal variants in BNIP3L gene.

Results: We performed targeted sequencing for all exons and un-translated regions of BNIP3L gene among 1806 patients with schizophrenia and 998 healthy controls of Han Chinese origin. Three rare nonsynonymous mutations, BNIP3L (NM_ 004331): c.52A>G, c.167G > A and c.313A>T, were identified in schizophrenia cases, and two of them were newly reported. The frequencies of these rare nonsynonymous mutations were significantly different between schizophrenia cases and healthy controls. For the common variants, rs147389989 achieved significance in both allelic and genotypic distributions with schizophrenia. Rs 1042992 and rs17310286 were significantly associated with schizophrenia in meta-analyses using PGC, CLOZUK, and our new datasets in this study.

Conclusions: Our findings provided further evidence that BNIP3L gene is a susceptibility gene of schizophrenia and revealed functional and potential causal mutations in BNIP3L. However, more functional validations are suggested to better understand the role of BNIP $3 L$ in the etiology of schizophrenia.
\end{abstract}

Keywords: BNIP3L gene, Schizophrenia, Targeted next-generation sequencing, Case-control study

\section{Background}

Schizophrenia is a severe mental disorder that affects about $1 \%$ of the world population. The core features of schizophrenia are positive symptoms including delusions and hallucinations, negative symptoms (especially impaired

\footnotetext{
*Correspondence: chenjhv@smhc.org.cn; shiyongyong@gmail.com †Juan Zhou and Chuanchuan Ma contributed equally to this work. ${ }^{3}$ Shanghai Clinical Research Center for Mental Health, Shanghai Key Laboratory of Psychotic Disorders, Shanghai Mental Health Center, Shanghai Jiao Tong University School of Medicine, Shanghai 200030, People's Republic of China

'Bio-X Institutes, Key Laboratory for the Genetics of Developmental and Neuropsychiatric Disorders (Ministry of Education), Collaborative Innovation Center for Brain Science, Shanghai Jiao Tong University, Shanghai 200030, People's Republic of China

Full list of author information is available at the end of the article
}

motivation, reduction in spontaneous speech, and social withdrawal) and cognitive impairment $[1,2]$. The first episode of schizophrenia usually occurs in late adolescence or early adulthood $[3,4]$. Around $20 \%$ of schizophrenia patients have chronic symptoms and disability, and over 50\% have intermittent but long-term psychiatric problems [5]. The etiology and pathogenesis of schizophrenia are not very clear, but genetic and environmental synergistic pathogeneses are generally accepted. The estimated heritability of schizophrenia is about $70-85 \%$ [6].

Schizophrenia is highly polygenic, as predicted by several genetic epidemiological researches many years ago [7]. Previous genome-wide association studies (GWASs) have revealed more than 100 distinct genetic loci are

(c) The Author(s). 2020 Open Access This article is licensed under a Creative Commons Attribution 4.0 International License, which permits use, sharing, adaptation, distribution and reproduction in any medium or format, as long as you give appropriate credit to the original author(s) and the source, provide a link to the Creative Commons licence, and indicate if changes were made. The images or other third party material in this article are included in the article's Creative Commons licence, unless indicated otherwise in a credit line to the material. If material is not included in the article's Creative Commons licence and your intended use is not permitted by statutory regulation or exceeds the permitted use, you will need to obtain permission directly from the copyright holder. To view a copy of this licence, visit http://creativecommons.org/licenses/by/4.0/ The Creative Commons Public Domain Dedication waiver (http://creativecommons.org/publicdomain/zero/1.0/) applies to the data made available in this article, unless otherwise stated in a credit line to the data. 
genome-wide associated with schizophrenia, which have en masse effects $[1,8,9]$. In 2017, Li et al. reported 30 new susceptibility loci of schizophrenia including rs73219805 locus near BNIP3L gene and predicting that the BNIP3L gene may be a susceptibility gene of schizophrenia [10]. Recent research about comparative genetic architectures of schizophrenia also revealed that intron variant of BNIP3L gene, rs117325001, was significantly associated with schizophrenia in a fixed-effect metaanalysis including individuals from East Asian and European ancestries [11].

The BNIP3L gene is located on chromosome $8 \mathrm{p} 21.2$ and harbors 6 exons, which encodes a 23.8-kDa protein called BCL2/adenovirus E1B $19 \mathrm{kDa}$ protein-interacting protein 3-like (NIP3L/NIX) and containing a transmembrane domain and a $\mathrm{BH} 3$ domain in the C-terminal region. NIP3L, which belongs to the NIP3 family, has two isoforms produced by alternative splicing (Fig. 1). The protein was a single-pass, transmembrane protein, located in the nuclear envelope, endoplasmic reticulum (ER) region, and outer mitochondrial membrane (OMM) [12, 13]. The transmembrane domain of NIP3L is glycine zipper, and every two NIP3L molecules form detergentresistant homodimers [12]. Ney stated that mitochondrial clearance in reticulocytes was affected by disruption of NIP3L's glycine zipper, which suggested that the function realization of NIP3L requires dimerization. The majority of NIP3L protein is cytoplasmic, which contains an LC3interacting region (LIR) motif and a novel short linear motif (SLiM) [12, 14]. LIR motif may recruit LC3 which bridges cargo with autophagy-generated membrane [15]. SLiM was inferred to interact with a hydrophobic pocket in another protein [12].

The functions of NIP3L mainly include inducing apoptosis and participating in mitochondrial quality control. On the one hand, NIP3L plays an important role in mitochondrial clearance. Previous researches have shown that a considerable proportion of erythrocytes had abnormally retained mitochondria in NIP3L-deficient mice while defective mitochondrial clearance appeared in NIXdeficient reticulocytes $[16,17]$. Some studies revealed that NIP3L may increase the production of mitochondrial reactive oxygen species (ROS) and competitively bind to BCL2 (or a related protein) liberating Beclin-1 from BCL2 complexes to activate autophagy [18]. On the other hand, NIP3L also regulates cell death [12]. NIP3L causes cytochrome $\mathrm{c}$ release to activate apoptosis by targeting to mitochondria triggers BAX/BAK-dependent mitochondrial outer membrane permeabilization (MOMP). NIP3L activates the classic mitochondrial permeability transition (MPT) to cause necrosis by increasing ER-SR (endoplasmic reticulum-sarcoplasmic reticulum) calcium stores and releasing calcium to mitochondria $[19,20]$.

From all above, NIP3L affects the normal metabolism and survival of cells through quality control of mitochondria. The hypothesis of mitochondrial dysfunction is one of the important pathogenic hypotheses of schizophrenia. Bahn et al. found that glycolysis, oxidative phosphorylation, and the tricarboxylic acid cycle (TCA cycle) were significantly downregulated in the brain tissue of schizophrenia patients [21]. Kung and Roberts have reported that, in postmortem human brain tissue of offdrug schizophrenics, the numbers of mitochondria per axon terminal appeared lower than on-drug schizophrenics or controls [22]. Schizophrenia has long been considered a neurodevelopment mental disorder [23]. The immuno-inflammatory pathway is also one of the widely appreciated mechanisms while abnormal clearance of depolarized mitochondria may cause an increase of ROS and trigger a set of inflammatory responses as well as abnormal density and dysfunction of mitochondria may affect neurodevelopment [24]. Synaptic transmission is another important neurobiological process, and the destruction of it may also be one of the important pathogenic factors of schizophrenia. Mitochondria play a pivotal role in assisting synaptic transmission at synapses [25]. Firstly, the process of synaptic transmission has a high demand for adenosine triphosphates

a.
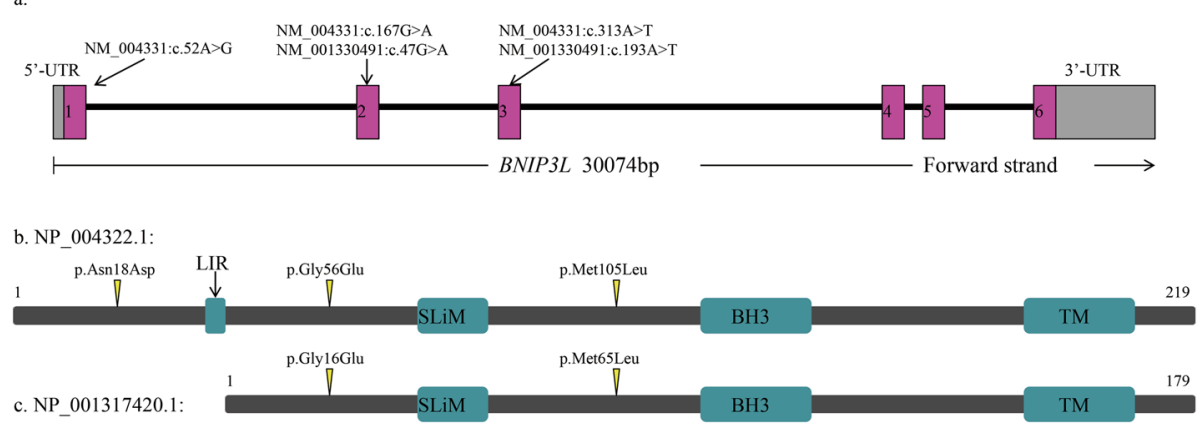

Fig. 1 Structure of BNIP3L gene and NIP3L protein. a BNIP3L gene structure, boxes 1 6 indicate the protein-coding exons. $\mathbf{b}$ NIP3L protein structure based on NP_0043221.1; TM is transmembrane domain. c NIP3L protein structure based on NP_001317420.1 
(ATPs) which are mainly provided by synaptic mitochondria [26, 27]. Secondly, mitochondria at synapses play a key role in maintaining the intra-synaptic calcium homeostasis [28] and assist the whole process of posttetanic potentiation which may be related to memory and behavior $[29,30]$. Thirdly, the synthesis, packaging, and enzymatic hydrolysis of some key neurotransmitters, such as acetylcholine, glutamate, dopamine, and serotonin, significantly rely on mitochondria [27].

All evidence shown above suggests that NIP3L may profoundly affect neuronal development through mitochondria and its biological functions probably be related to the pathogenesis of schizophrenia. In this study, we performed targeted next-generation sequencing for all exons and un-translated region (UTR) of BNIP3L gene in 1806 patients with schizophrenia and 998 healthy controls to identify potential pathogenic mutations of $B N I P 3 L$ gene in schizophrenia.

\section{Methods}

\section{Subjects}

The sample set included 1806 unrelated schizophrenia cases (1111 men and 695 women) and 998 independent healthy controls (437 men and 561 women). The mean age is 44.49 years (standard deviation $=12.13$ ) among schizophrenia cases and 43.15 years (standard deviation $=17.53$ ) among healthy controls (Table 1 ).

The study was approved by the ethical committee. All participants were recruited from Han Chinese population and signed informed consent. All patients were diagnosed by two independent psychiatrists from the Affiliated Wuxi Mental Health Center with Nanjing Medical University. Diagnoses were made strictly according to the DSM-IV criteria based on SCID-I (the Structured Clinical Interview for DSM-IV Axis I Disorders). Patients were excluded if they had suffered mood disorder, neurological illness, mental retardation, psychotic disorder, and history of substance use due to general medical conditions. During the physical examination, healthy controls were collected.

\section{DNA extraction, target capture, and next-generation sequencing}

LifeFeng Genomic DNA Purification Kits (Lifefeng Biotech Co., Ltd., Shanghai, China) were used to extract genomic DNA from peripheral blood samples. DNA

Table 1 Characteristics of the study sample set

\begin{tabular}{lllllll}
\hline & $n$ & & & & \multicolumn{2}{l}{ Age, years } \\
\cline { 2 - 3 } & Men & Women & Total & & Mean & s.d. \\
\hline Patients with SCZ & 1111 & 695 & 1806 & & 44.49 & 12.13 \\
Healthy controls & 437 & 561 & 998 & & 43.15 & 17.53 \\
\hline
\end{tabular}

SCZ schizophrenia, s.d. standard deviation concentration and quality were examined by NanoDrop2000 (Thermo Scientific, USA). Twenty-three pairs of primers divided into two pools were designed covering all exons, UTRs, and exon-intron boundary of $B N I P 3 L$ gene. The sequences of primers and their targeted regions are shown in Table S1. Two-staged PCR process was performed for library construction. All the PCR reagents and protocol were supported by Shanghai DYnastyGene Company. High Sensitivity DNA kits and 2100 Bioanalyzer (Agilent Technologies, USA) were used to determine the size distribution of DNA library fragments. The Illumina HiSeq X Ten System (Illumina, USA) was used to sequence the final DNA libraries as PE 150 bp reads.

\section{Variant identification and validation}

Genome Analysis Toolkit (GATK) Best Practices [31], the pipeline for germline short variant discovery, was run on every data set independently. Burrows-Wheeler Aligner (BWA) [32] was used to align raw reads to the human reference genome (GRCh38), GATK haplotypercaller was used to call variants (single-nucleotide polymorphisms, SNPs; short insertions and deletions, InDels), and Annovar [33] was used to annotate the variants. Multi-species alignments were performed using Clustal Omega online software [34]. Sanger sequencing was performed to verify the rare nonsynonymous variants.

\section{Case-control study}

Single locus association tests, Hardy-Weinberg equilibrium (HWE), and pairwise linkage disequilibrium (LD) analysis were performed on the SHEsisPlus online software platform [35-37]. For the common variants, pairwise LD analysis was performed and adjacent loci with $\mathrm{D}^{\prime}>0.90$ were classified in the same block. The chisquare test and Fisher's exact test were used to infer whether polymorphisms of the alleles and genotypes were associated with schizophrenia. Fisher's exact test would be used if the theoretical frequency less than five occurred in any group. All tests were two-tailed, and statistical significance was set at $P<0.05$. The false discovery rate (FDR-BH) was used to calibrate the $P$ values.

\section{Meta-analyses}

CLOZUK and Independent PGC2 datasets were used for the meta-analysis with our data. We performed metaanalyses by RevMan5.3. Heterogeneity analysis of the odds ratios was tested by Cochran's Q test and $I^{2}$. No significant heterogeneity existed, and fixed effects model was used when $P>0.05$ and $I^{2}<50 \%$; otherwise, random effects model would be selected [38]. 


\section{Results}

\section{Variants identification}

We performed Sanger sequencing validation for ten samples containing missense mutations detected by next-generation sequencing. Among them, the three mutations of eight samples were verified to be true positives, and their next-generation sequencing depths are all deeper than $20 \times$. The other 2 samples were verified to be false positives, and their depths were all lower than $10 \times$. Therefore, only the variants with a depth more than $10 \times$ were included in further analysis.

A total of 114 high-quality variants were identified in case and control groups including six variants in coding exons, 25 intron variants, one upstream variant, and 82 UTR variants (Table S2). Three databases, the $1000 \mathrm{Ge}-$ nomes Project [39], the Exome Aggregation Consortium [40], and NHLBI Exome Sequencing Project [41] were used to filter rare mutations. Only loci with minor allele frequency (MAF) less than 0.01 in each database were selected, and in total, 105 rare mutations including 69 novel variants were detected. The other nine variants are common SNPs, and their information is shown in Table S3.

\section{Analyses of rare variants in coding regions}

In total, six variants in coding exons including two synonymous mutations, one nonframeshift deletion, and three nonsynonymous mutations (Fig. 1) were identified.
They are all heterozygous and rare mutations. Among them, only one synonymous mutation was detected from one sample of the control group. Other mutations were all from the case group and were detected in 12 independent patients, of which eight patients were with the nonsynonymous mutations. We performed Sanger sequencing to verify all the nonsynonymous variants, BNIP3L (NM_ 004331): c.52A $>$ G, c.167G $>A$ and c.313A $>$ T. The results are shown in Fig. 2. All variants were verified to be heterozygous mutations. In addition, c.167G $>\mathrm{A}$ and c.313A>T were newly reported.

PolyPhen-2 [42], MutationTaster [43], SIFT [44], and LRT [45] in Annovar were used to predict the pathogenicity of the variants (Table 2). All the three variants are predicted to be "disease causing" by MutationTaster. By SIFT, c.52A $>\mathrm{G}$ and c.167G $>\mathrm{A}$ were predicted to be deleterious and c.313A $>\mathrm{T}$ to be tolerated. By LRT, c.167G $>\mathrm{A}$ and c. $313 \mathrm{~A}>\mathrm{T}$ were predicted to be deleterious as well as c. $52 \mathrm{~A}>\mathrm{G}$ to be neutral. Moreover, by Polyphen-2, c.52A $>\mathrm{G}$ was predicted to be possibly damaging, c.167G $>A$ to be probably damaging, and c.313A $>\mathrm{T}$ to be benign respectively. We also access the CADD (Combined Annotation Dependent Depletion) score [46] of the three variants, and their scores are all greater than 20, which means that the variants are among the top $1 \%$ of deleterious variants in the human genome.

The amino acid variants for the three rare nonsynonymous mutations are BNIP3L (NP_004322): p.Asn18Asp,

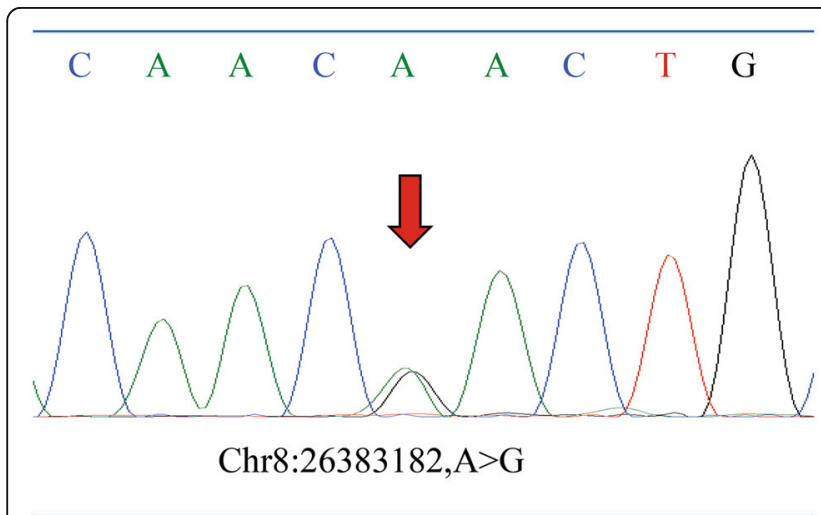

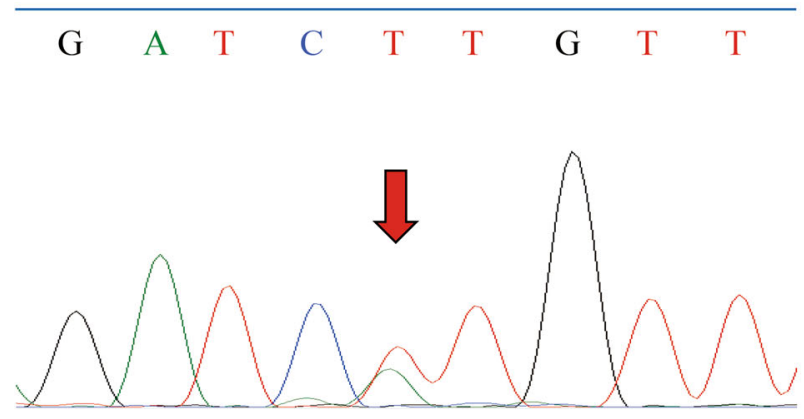

Chr8:26395258,A $>$ T

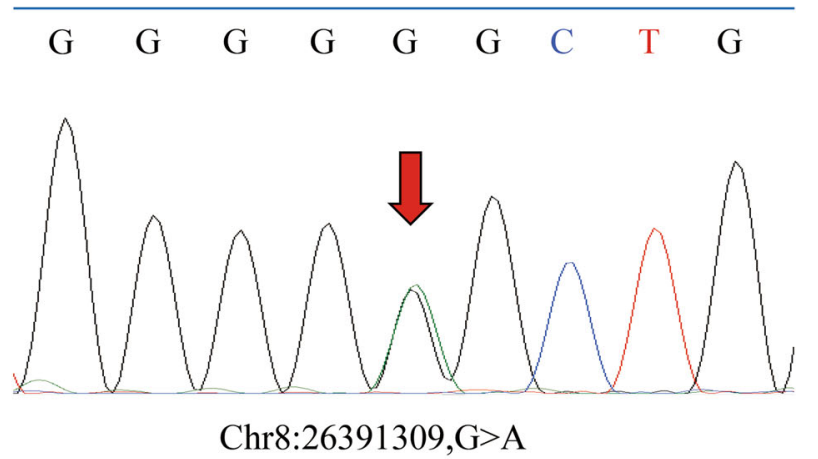

A $\begin{array}{llllllllll}\text { A } & \text { A } & \text { A } & \text { G } & \text { G } & \text { G } & \text { G } & \text { G } & \text { C }\end{array}$
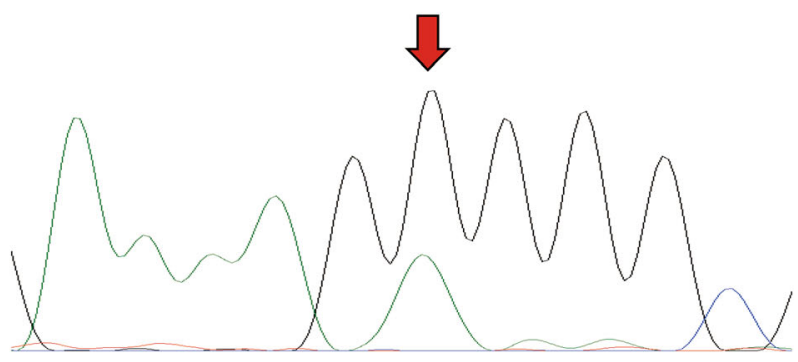

Chr8: 26383046,G>A

Fig. 2 The results of Sanger sequence verifying the rare nonsynonymous variants. Arrows indicate the mutation sites 
Table 2 Detailed information of rare mutations detected in this study

\begin{tabular}{|c|c|c|c|c|c|c|c|c|c|c|c|}
\hline Variants & Position & $\begin{array}{l}\text { Variants } \\
\text { status }\end{array}$ & SIFT & Polyphen-2 & MutationTaster & LRT & $\begin{array}{l}\text { CADD } \\
\text { score }\end{array}$ & $\begin{array}{l}\text { Novel or } \\
\text { not }\end{array}$ & Individuals & Gender & Group \\
\hline \multirow{5}{*}{$\begin{array}{l}\text { NM_004331: c.52A>G/ } \\
\text { p.Asn18Asp }\end{array}$} & \multirow[t]{5}{*}{ chr8:26383182 } & \multirow[t]{5}{*}{ Heterozygous } & \multirow[t]{5}{*}{$\mathrm{D}$} & \multirow[t]{5}{*}{$P$} & \multirow[t]{5}{*}{$\mathrm{D}$} & \multirow[t]{5}{*}{$\mathrm{N}$} & \multirow[t]{5}{*}{23.6} & \multirow[t]{5}{*}{ rs549425213 } & 5550002 & \multirow[t]{3}{*}{ Male } & \multirow[t]{5}{*}{ SCZ } \\
\hline & & & & & & & & & 5550703 & & \\
\hline & & & & & & & & & DI226 & & \\
\hline & & & & & & & & & $55 \$ 0439$ & \multirow[t]{2}{*}{ Female } & \\
\hline & & & & & & & & & DI182 & & \\
\hline $\begin{array}{l}\text { NM_004331: c.167G>A } \\
\text { p.Gly56Glu, NM_001330491: } \\
\text { c.47G>A/p.Gly16Glu }\end{array}$ & chr8:26391309 & Heterozygous & D & D & D & D & 31 & novel & YZ005 & Male & SCZ \\
\hline \multirow{2}{*}{$\begin{array}{l}\text { NM_004331: c.313A>T/ } \\
\text { p.Met105Leu, } \\
\text { NM_001330491: c.193A>T/ } \\
\text { p.Met65Leu }\end{array}$} & \multirow[t]{2}{*}{ chr8:26395258 } & \multirow[t]{2}{*}{ Heterozygous } & \multirow[t]{2}{*}{ T } & \multirow[t]{2}{*}{ B } & \multirow[t]{2}{*}{ D } & \multirow[t]{2}{*}{ D } & \multirow[t]{2}{*}{22.1} & \multirow[t]{2}{*}{ novel } & SYS0036 & \multirow[t]{2}{*}{ Female } & \multirow[t]{2}{*}{ SCZ } \\
\hline & & & & & & & & & DI010 & & \\
\hline
\end{tabular}

$D$ deleterious for SIFT and LRT, probably damaging for Polyphen-2, disease_causing for MutationTaster; $T$, tolerated; $P$, possibly damaging; $B$, benign; $N$, neutral; SCZ, schizophrenia; CADD, Combined Annotation Dependent Depletion

p.Gly56Glu, and p.Met105Leu. Multiple alignments of NIP3L protein sequences from several available species showed that p.Gly56Glu is located in a highly conserved region evolutionally while the other two loci, p.Asn18Asp and p.Met105Leu, are not so that conserved (Fig. 3).

\section{Association analysis of all variants}

Case-control study was performed for all the variants except for four single-nucleotide variants (SNVs) including one rare and three common variants, because these four SNVs did not reach Hardy-Weinberg equilibrium in the healthy controls. No significant association was observed between rare variants and schizophrenia in our sample set. Although the rare variant, rs549425213 ( $P_{\text {allele }}=0.096$ before correction $)$, and our novel reported variant in 5' ${ }^{\prime}$-UTR, NM_004331:C.-85G $>$ A $\left(P_{\text {allele }}=0.068\right.$ before correction), are not significantly associated with schizophrenia, the variants are only detected in cases and their $p$ values are very close to 0.05 . We also performed gene-based association study for nonsynonymous mutations. In total, eight patients with schizophrenia carried nonsynonymous mutations which were not detected in the control group. The chi-square test was performed, and the accumulated number of nonsynonymous mutation carriers in the case group and control group was significantly different $\left(\mathrm{chi}^{2}=4.433, P\right.$ $=0.035$ ).

The results for common variants are shown in Table 3. Rs147389989 achieved both allelic and genotypic significance after FDR-BH correction $\left(P_{\text {allele }}=\right.$ $0.007, P_{\text {genotype }}=0.017$, odds ratio $(\mathrm{OR})$ for “G” $[95 \%$ confidence interval $(\mathrm{CI})]=2.383[1.536 \sim 3.698])$, and the allele " $G$ " was suggested to be a risk factor for schizophrenia. Before FDR-BH correction, rs1055476 $\left(P_{\text {allele }}=0.004, P_{\text {genotype }}=0.004\right.$, OR for “-" $[95 \% \mathrm{CI}]$ $=3.245[1.362 \sim 7.728])$ and $\operatorname{rs} 17310286\left(P_{\text {allele }}=0.012\right.$,

Xenopus_tropicalis
Cattle
Mouse
Human
Macaca_mulatta
Cricetulus_griseus
Cervus_elaphus_hippelaphus

Xenopus tropicalis

Cattle

Mouse

Human

Macaca mulatta

Cricetulus griseus

Cervus_elaphus_hippelaphus

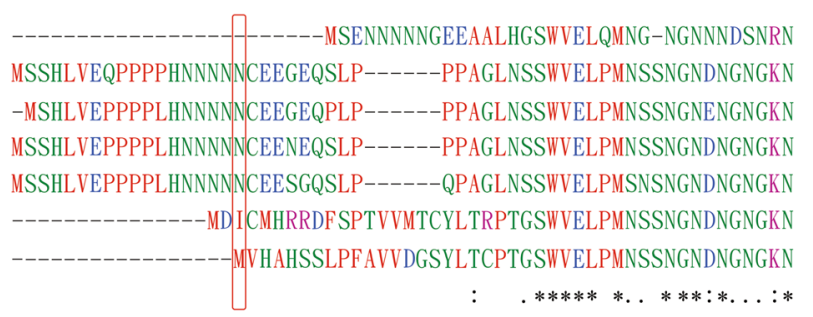

35

54

53

54

54

45

43

GG̈LEHVPSSSSI HDGDNEKILLDAQHESGQSSSRSSSQCDSPSP-EDGQITPDVVENHTSK GGLEHVPSSSS I HNGDMEK ILLDAQHESGQSSSRGSSHCDSPSPQEDGQ IMFDVEMHTSK GGLEHVPSSSS I HNGDMEK ILLDAQHESGQSSSRGSSHCDSPSPQEDGQ IMFDVEMHTSR GGLEHVPSSSSI HNGDMEK ILLDAQHESGQSSSRGSSHCDSPSPQEDGQIMFDVEMHTSR GGLEHVPSSSS IHNGDVEK ILLDAQHESGQSSSRGSSHCDSPSPQEDGQ IMFDVEVHTSR GGLEHVPSSSS IHNGDNEK ILLDAQHESGQSSSRGSSHCDSPSPQEDGQIMFDVENHTSR GGLEHVPSSSS I HNGDNEK ILLDAQHESGQSSSRGSSHCDSPSPQEDGQIMFDVENHTSK

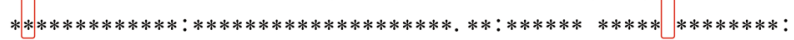

94

114 113 114 114 105 103

Fig. 3 Multiple alignments of NIP3L protein sequences of various species. The sites of nonsynonymous variants were box out 


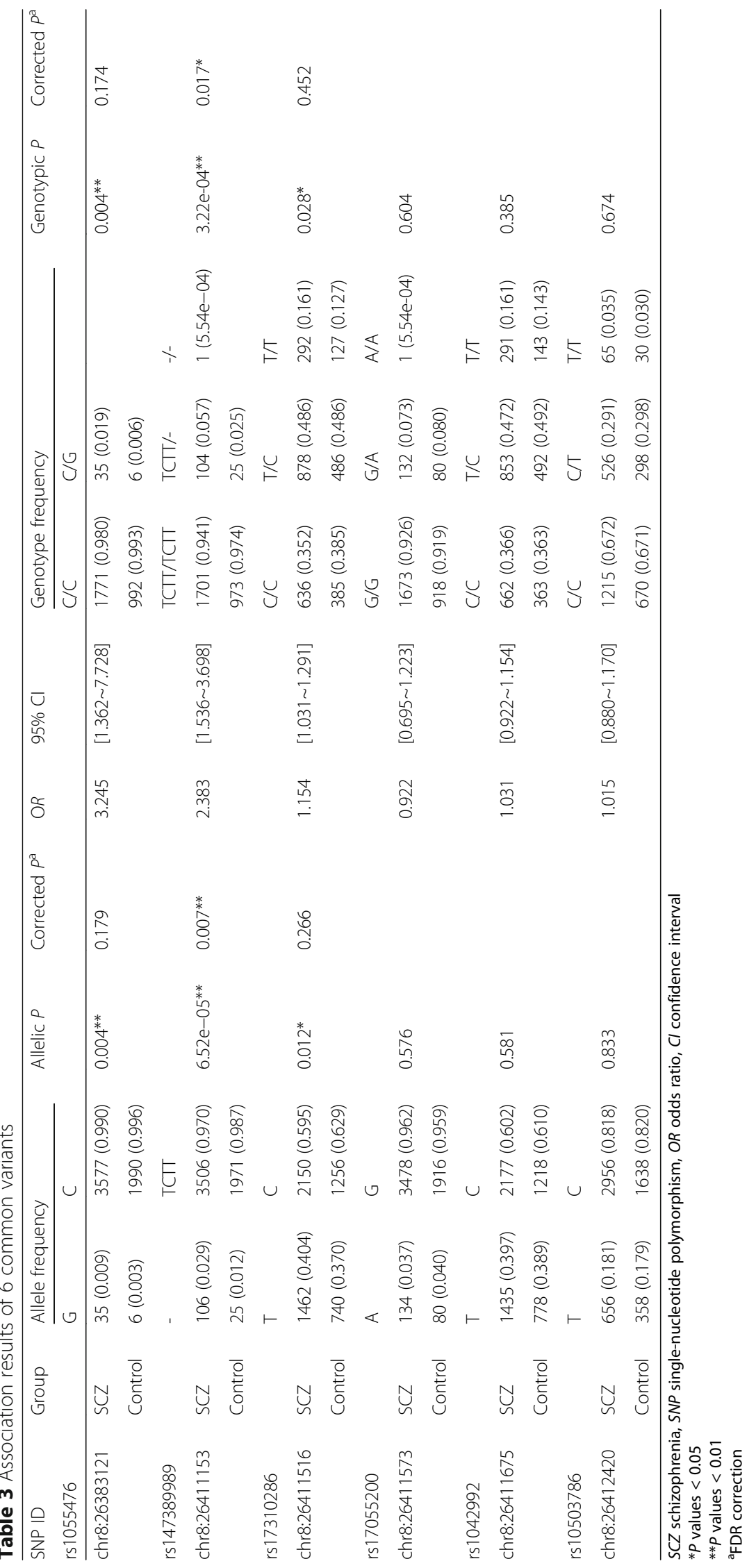


$P_{\text {genotype }}=0.028$, OR for “T" $[95 \% \mathrm{CI}]=1.154$ [1.031 1.291]) were significantly associated with schizophrenia both in allelic and genotypic distributions, but there was no significance after correction.

Pairwise LD analysis indicated that all the six common variants formed one haplotype block (Fig. 4). With frequency > 0.03, four haplotypes, C-TCTT-C-G-C-C/CTCTT-T-G-T-C/C-TCTT-C-G-C-T/C-TCTT-C-A-C-C, were identified. Haplotype "C-TCTT-T-G-T-C" was implied to be a significant risk factor for schizophrenia $(P$ $=5.95 \times 10^{-4}$ after FDR correction, OR $[95 \% \mathrm{CI}]=1.225$ [1.093 1.373]). The global Pearson's $p$ is 0.048 (Table 4).

\section{The results of meta-analyses}

From the results of previous GWASs [8, 9] and SZDB2.0 database $[47,48]$, rs17310286 and rs1042992 were genotyped before. Therefore, the results of previous GWAS and our data were used for meta-analysis (Table 5). CLOZUK and independent PGC datasets were used for meta-analyses with our results. No heterogeneity was detected in the combined sample sets (rs17310286: $I^{2}=$ $31 \%$, $\mathrm{chi}^{2}=1.45, P=0.23$; rs1042992: $I^{2}=0 \%$, chi $^{2}=$ $1.55, P=0.46)$, so fixed effects model was chosen. Although these two loci were not significantly associated with schizophrenia in our sample set, significant associations were observed after meta-analyses (rs17310286: pooled OR $[95 \% \mathrm{CI}]=0.93[0.91-0.95], Z=6.25$, and $P<$ $1.00 \times 10^{-4} ;$ rs1042992: pooled OR $[95 \% \mathrm{CI}]=0.93[0.91-$ 0.95], $Z=5.92$, and $P<1.00 \times 10^{-4}$ ) as shown in forest plot of Figs. 5 and 6. The alternate allele "T" for the both two loci were suggested to be risk factors for schizophrenia which was consistent with the results of previous GWAS research [9].

\section{Discussion}

In our results, we found three rare nonsynonymous mutations in the BNIP3L gene, c.52A $>\mathrm{G}$, c.167G $>\mathrm{A}$ and c.313A $>$ T, carried by eight patients with schizophrenia. Among them, c.167G $>\mathrm{A}$ and c.313A $>\mathrm{T}$ were newly reported and all of them were predicted to be diseasecausing variants by MutationTaster. All three mutations are located in the extra-membrane region of the NIP3L protein, suggesting that the variants may affect the interaction of NIP3L with other proteins and may play a key role in the pathogenesis of schizophrenia. Our novel reported variant in 5'-UTR, NM_004331: c. $-85 \mathrm{G}>\mathrm{A}$, is carried by six patients with schizophrenia which is not detected in the control group. We also performed the Sanger sequencing to verify BNIP3L (NM_004331): c.-85G>A, and the results are shown in Fig. 2. In the case DI226, heterozygous c.$85 \mathrm{G}>\mathrm{A}$ and c.52A $>\mathrm{G}$ (nonsynonymous mutations) were detected as bi-allelic mutations for schizophrenia. The CADD score of c. $-85 G>A$ is 20.6 , and it is predicted to be "disease causing" by MutationTaster. As predicted by MutationTaster, c. $-85 \mathrm{G}>\mathrm{A}$ might cause splice site changes and affect protein features. The results of the gene-based association analysis

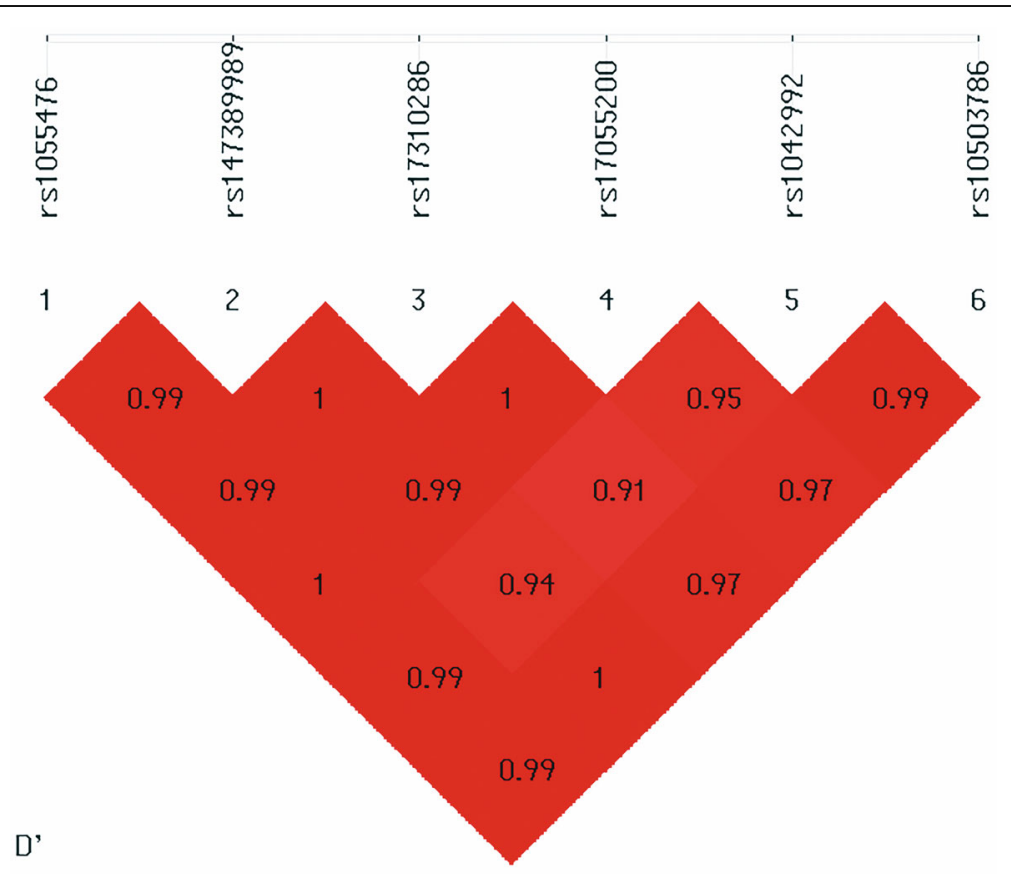

Fig. 4 Pairwise linkage disequilibrium plot for the common variants in BNIP3L gene. The pairwise $D^{\prime}$ values are presented in the matrices; deep red implicates relatively strong linkage disequilibrium and vice versa 
Table 4 Results of haplotype analysis

\begin{tabular}{lllllllll}
\hline Haplotype $^{\mathrm{a}}$ & Case frequency & Control frequency & Chi $^{2}$ & $P$ & ${\text { Corrected } P^{\mathrm{b}}}$ & OR [95\% Cl] & Global Chi $^{2}$ & Global $P$ \\
\hline C-TCTT-C-G-C-C & $1209(0.334)$ & $707(0.354)$ & 2.171 & 0.140 & 0.162 & $0.917[0.817 \sim 1.028]$ & 7.904 & $0.048^{*}$ \\
C-TCTT-T-G-T-C & $1408(0.389)$ & $684(0.342)$ & 12.207 & $4.76 \mathrm{e}-04^{* *}$ & $5.95 \mathrm{e}-04^{* *}$ & $1.225[1.093 \sim 1.373]$ & \\
C-TCTT-C-G-C-T & $643(0.178)$ & $349(0.174)$ & 0.088 & 0.765 & 0.820 & $1.022[0.885 \sim 1.179]$ & \\
C-TCTT-C-A-C-C & $131(0.036)$ & $72(0.036)$ & 0.001 & 0.970 & 0.970 & $1.005[0.750 \sim 1.347]$ & \\
\hline
\end{tabular}

$O R$ odds ratio, $\mathrm{Cl}$ confidence interval

${ }^{*} P$ values $<0.05$

** $P$ values $<0.01$

a Haplotypes with frequency $<0.03$ were ignored

${ }^{\mathrm{b}} \mathrm{FDR}$ correction

showed that the number of nonsynonymous mutations carried by schizophrenia patients was significantly more than healthy controls, which further confirmed that the BNIP3L gene is a susceptibility gene of schizophrenia.

We found rs147389989 in the BNIP3L gene was significantly associated with schizophrenia, and we searched for this locus in the SZDB database [47]. We found that rs147389989 has been genotyped in schizophrenia cases and healthy controls in CLOZUK sample sets [9]. But they did not observe any significant association between rs147389989 and schizophrenia. In our study, all participants were recruited from Han Chinese population while CLOZUK samples were from European, and this may cause the inconsistency between our results and previous study. The recent genome-wide association study [9] of schizophrenia identified 145 associated loci through meta-analysis, and $r$ rs1042992 $(P=3.67$ $\times 10^{-9}$, OR for allele " $C$ " $=0.93$, standard error $(\mathrm{SE})$ $=0.012)$ is one of the 50 novel associated loci. Moreover, rs17310286, which highly linked with rs1042992, was also significantly associated with schizophrenia $\left(P=5.43 \times 10^{-9}\right.$, OR for allele " $\mathrm{C}$ " = $0.93, \mathrm{SE}=0.012)$. But in our results, the significant association between rs1042992 and schizophrenia was not observed. Although rs17310286 was significantly associated with schizophrenia both in allelic and genotypic distributions, there was no significance after correction. So we performed metaanalyses using the results of the GWAS and our study. No heterogeneity was detected in the combined sample sets. These two loci were both significantly associated with schizophrenia in meta-analyses and had the same direction of effect with the previous GWAS. Our negative results of these two loci may be caused by the limited size of our sample set. From the results of haplotype analysis of our data, we found that the haplotype, C-TCTT-T-G-T-C, was a significant risk factor for schizophrenia and the two loci, rs1042992 and rs17310286, were both the risk allele " $\mathrm{T}$ " for the haplotype which was consistent with the results of meta-analyses. The results of meta-analyses and the haplotype analysis further confirmed that rs1042992 and rs17310286 located in 3'-UTR of BNIP3L gene were significantly associated with schizophrenia and provided more evidence for BNIP3L gene as a candidate gene for schizophrenia.

The three common variants significantly associated with schizophrenia in our study are located in the 3 'UTR of the BNIP3L gene. It is suggested that the $3^{\prime}$ UTR of the BNIP3L gene may play a crucial role in schizophrenia. 3'-UTR is a pivotal region which regulates gene expression, protein-protein interactions, and

Table 5 Results of meta-analysis

\begin{tabular}{|c|c|c|c|c|c|c|c|c|c|c|c|}
\hline Variants & Allele & Data set & OR & SE & $P$ value & Model & Heterogeneity $P^{2}$ & $\begin{array}{l}\text { Heterogeneity } \\
P \text { value }\end{array}$ & Total OR [95\%Cl] & $\begin{array}{l}\text { Overall } \\
\text { Effect (Z) }\end{array}$ & $\begin{array}{l}\text { Total } \\
P \text { value }\end{array}$ \\
\hline rs17310286 & $\mathrm{C} / \mathrm{T}$ & CLOZUK+PGC2 & 0.930 & 0.012 & $5.43 e-09^{* *}$ & Fixed & $31 \%$ & 0.23 & $0.93[0.91 \sim 0.95]$ & 6.25 & $P<1.00 \mathrm{e}-05^{* *}$ \\
\hline chr8:26411516 & & Our Study & 0.867 & 0.057 & $0.012^{*}$ & & & & & & \\
\hline rs1042992 & $\mathrm{C} / \mathrm{T}$ & Independent PGC2 & 0.937 & 0.015 & $1.94 \mathrm{e}-05^{* *}$ & Fixed & $0 \%$ & 0.46 & $0.93[0.91 \sim 0.95]$ & 5.92 & $P<1.00 \mathrm{e}-05^{* *}$ \\
\hline \multirow[t]{2}{*}{ chr8:26411675 } & & CLOZUK & 0.912 & 0.022 & $2.79 \mathrm{e}-05^{* *}$ & & & & & & \\
\hline & & Our Study & 0.970 & 0.057 & 0.581 & & & & & & \\
\hline
\end{tabular}

Our study: Han Chinese population

$O R$ odds ratio, $\mathrm{Cl}$ confidence interval

${ }^{*} P$ values $<0.05$

**P values $<0.01$ 


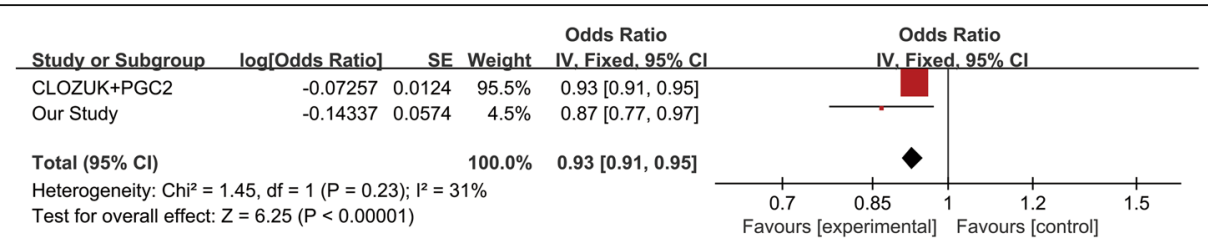

Fig. 5 Forest plot of meta-analysis result for rs17310286

mRNA localization by binding with micro-RNAs (miRNAs) or RNA-binding proteins (RBPs) $[49,50]$. Several studies have reported that some SNPs affect the risk of schizophrenia because their alter alleles can change the binding stability of some miRNAs with the 3'-UTR of the gene [51-53]. Dysregulation of some miRNAs is associated with central nervous system diseases including schizophrenia [54].

In our study, for rs1042992 and rs17310286, we used SNPinfo Web Server [55] to predict their possible functions. We found that the allele "T" of rs1042992 would create the binding of hsa-miR-495 and hsa-miR-590-3p with the 3'-UTR. Interestingly, previous research has shown that hsa-miR-495 was one of the human miRNAs that were significantly differentially expressed $(P<0.05)$ between autistic and nonautistic individuals [56]. HsamiR-495 was also identified to involve in mechanisms to enhance neuroprotection [57] and selectively affect addiction-related behaviors [58]. Moreover, hsa-miR590-3p was proven to involve in mitochondrial dysfunction and oxidative stress and play a key role in the pathogenesis of Parkinson's disease [59]. All of the above suggested that the polymorphism of rs1042992 and rs17310286 might regulate the expression of NIP3L by changing some miRNAs binding and affect the risk of schizophrenia.

Our study identified several variants in BNIP3L gene that may affect the expression and function of NIX, while the role of NIX in mitochondrial clearance and apoptosis has been verified which is important for neuron protection in brain. Yuan et al. revealed the involvement of NIX in cerebral ischemia-reperfusion (IR)-induced mitophagy which could attenuate brain injury after cerebral ischemia. Their research found that Bnip3l knockout (bnip3l-/-) impaired mitophagy and aggravated cerebral I-R injury in mice, which can be rescued by NIX overexpression [60]. Ma et al. reported that in a rat traumatic brain injury model, NIX expression colocalized with neuronal cells in cortical areas. Moreover, autophagy increased and neuronal apoptosis decreased after inducing upregulation of NIX. They suggested that NIX probably plays a neuroprotective role in rat traumatic brain injury through autophagy and apoptosis pathways [61]. Therefore, BNIP3L may protect neurons through mitochondrial quality control and may be related to the pathogenesis of some mental diseases.

Our results provided additional evidence that BNIP3L was a susceptibility gene of schizophrenia. However, there are two limitations of our study. One is the limited sample size which may be the reason why no significant association between single rare nonsynonymous variants and schizophrenia was observed. And the other is the lack of functional verification. Targeted sequencing for BNIP3L gene among more patients with schizophrenia and more functional validations are considered to be necessary for further understanding the etiology correlated with BNIP3L in schizophrenia.

\section{Conclusions}

Our research presented a comprehensive mutation spectrum of BNIP3L gene in schizophrenia. In $B N I P 3 L$, we found that the number of total rare nonsynonymous mutation carriers in schizophrenia cases and healthy controls were significantly different. For the common variants, we found rs147389989 was significantly associated with schizophrenia in Han Chinese population. By meta-analyses, we also confirmed rs1042992 and rs17310286 were both significantly associated with schizophrenia. All the results further confirmed that BNIP3L gene is a susceptibility gene of schizophrenia, but further functional validations

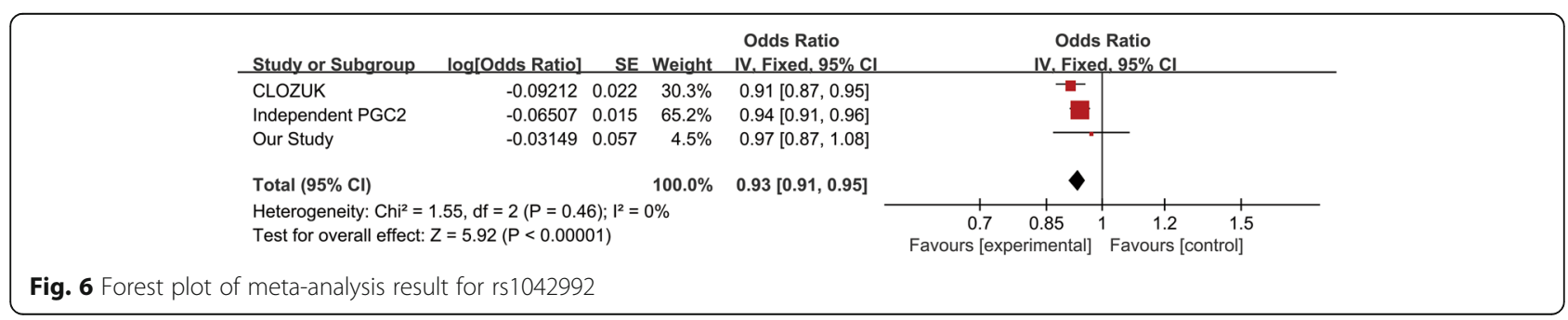


are needed for understanding the role of $B N I P 3 L$ in the pathogenesis of schizophrenia.

\section{Supplementary information}

Supplementary information accompanies this paper at https://doi.org/10. 1186/s40246-020-00266-4.

Additional file 1: Table S1. The sequences of primers and their targeted regions. Table S2. A total of 114 high-quality variants were identified in case and control groups. Table S3. Detailed information of the 9 common variants in the BNIP3L Gene.

\section{Abbreviations}

SNP: Single nucleotide polymorphism; GWASs: Genome-wide association studies; ER: Endoplasmic reticulum; OMM: Outer mitochondrial membrane; LIR: LC3-interacting region; SLiM: Short linear motif; ROS: Reactive oxygen species; MOMP: Mitochondrial outer membrane permeabilization; MPT: Mitochondrial permeability transition; SR: Sarcoplasmic reticulum; TCA cycle: The tricarboxylic acid cycle; ATPs: Adenosine triphosphates; UTR: Untranslated region; GATK: Genome Analysis Toolkit; BWA: Burrows-Wheeler Aligner; HWE: Hardy-Weinberg equilibrium; LD: Linkage disequilibrium; FDR$\mathrm{BH}$ : False discovery rate; MAF: Minor allele frequency; CADD: Combined Annotation Dependent Depletion; SNVs: Single nucleotide variants; OR: Odds ratio; Cl: Confidence interval; SE: Standard error; miRNAs: Micro-RNAs; RBPS: RNA-binding proteins; I-R: Ischemia-reperfusion

\section{Acknowledgements}

We thank all of patients and healthy controls who were involved in this study. And we are grateful for the help from psychiatrists during recruitment and diagnosis of schizophrenia patients.

\section{Authors' contributions}

Y.S. and J.C. designed and supervised the whole research process. J.Z. and C.M. carried out all experiments and managed the literature searches. H.Z. and X.L. conducted DNA extraction and Sanger sequencing. K.W. undertook the statistical analysis. X.J. was responsible for platform coordination and management. J.Yuan and J.Yin conducted patient recruitment and diagnosis as well as sample collection. J.Z. wrote the first draft of the manuscript. J.C. and Y.S. proofread the article. All authors contributed to and approved the final manuscript.

\section{Funding}

This work was supported in part by Shanghai Municipal Science and Technology Major Project (Grant No. 2017SHZDZX01), the National Key R\&D Program of China (2017YFC0908105, 2019YFA0905401 and 2016YFC1306900), the Natural Science Foundation of China (U1804284, 81421061, 81701321, 31571012, 91849104, 31770800, 81571329, 81501154 and 81871051), Shanghai Hospital Development Center (SHDC12016115), Shanghai Science and Technology Committee (17JC1402900 and 17490712200), and Shanghai Municipal Health Commission (ZK2015B01 and 201540114). The funding bodies did not participate in the design of the study; in collection, analysis, and interpretation of data; or in writing the manuscript.

\section{Availability of data and materials}

All data generated or analyzed during this study are included in this published article and its supplementary information files.

\section{Ethics approval and consent to participate}

All participants signed informed consent, and the study was approved by the ethical committee of Bio-X Institutes, Shanghai Jiao Tong University. The reference number is 20190918 .

\section{Consent for publication}

Not applicable.

\section{Competing interests}

The authors declare that they have no competing interests.

\section{Author details}

${ }^{1}$ Bio-X Institutes, Key Laboratory for the Genetics of Developmental and Neuropsychiatric Disorders (Ministry of Education), Collaborative Innovation Center for Brain Science, Shanghai Jiao Tong University, Shanghai 200030, People's Republic of China. ${ }^{2}$ Brain Science Basic Laboratory, The Affiliated Wuxi Mental Health Center With Nanjing Medical University, Wuxi, 21415 Jiangsu Province, People's Republic of China. ${ }^{3}$ Shanghai Clinical Research Center for Mental Health, Shanghai Key Laboratory of Psychotic Disorders, Shanghai Mental Health Center, Shanghai Jiao Tong University School of Medicine, Shanghai 200030, People's Republic of China. ${ }^{4}$ Department of Psychological Medicine, Institute of Psychiatry, Psychology \& Neuroscience, King's College London, London, UK.

Received: 31 January 2020 Accepted: 24 April 2020

Published online: 11 May 2020

\section{References}

1. Owen MJ, Sawa A, Mortensen PB. Schizophrenia. Lancet. 2016;388(10039): 86-97.

2. Joyce EM, Roiser JP. Cognitive heterogeneity in schizophrenia. Curr Opin Psychiatry. 2007;20(3):268-72

3. Lieberman JA, Perkins D, Belger A, Chakos M, Jarskog F, Boteva K, et al. The early stages of schizophrenia: speculations on pathogenesis, pathophysiology, and therapeutic approaches. Biol Psychiatry. 2001:50(11): 884-97.

4. Addington J, Heinssen R. Prediction and prevention of psychosis in youth at clinical high risk. Annu Rev Clin Psychol. 2012;8(1):269-89.

5. Barbato A. Psychiatry in transition: outcomes of mental health policy shift in Italy. Aust N Z J Psychiatry. 1998;32(5):673-9.

6. Burmeister M, McInnis MG, Zöllner S. Psychiatric genetics: progress amid controversy. Nat Rev Genet. 2008:9:527-40.

7. Gottesman II, Shields J. A polygenic theory of schizophrenia. Proc Natl Acad Sci U S A. 1967:58(1):199-205.

8. Ripke S, Neale BM, Corvin A, Walters JTR, Farh KH, Holmans PA, et al. Biological insights from 108 schizophrenia-associated genetic loci. Nature. 2014;511(7510):421-7.

9. Pardiñas AF, Holmans P, Pocklington AJ, Escott-Price V, Ripke S, Carrera N, et al. Common schizophrenia alleles are enriched in mutation-intolerant genes and in regions under strong background selection. Nat Genet. 2018; 50(3):381-9.

10. Li Z, Chen J, Yu H, He L, Xu Y, Zhang D, et al. Genome-wide association analysis identifies 30 new susceptibility loci for schizophrenia. Nat Genet. 2017:49:1576-83.

11. Lam M, Chen C-Y, Li Z, Martin AR, Bryois J, Ma X, et al. Comparative genetic architectures of schizophrenia in east Asian and European populations. Nat Genet. 2019:51(12):1670-8.

12. Ney PA. Mitochondrial autophagy: origins, significance, and role of BNIP3 and NIX. Biochim Biophys Acta. 2015;1853(10, Part B):2775-83.

13. Chen G, Cizeau J, Vande Velde C, Park JH, Bozek G, Bolton J, et al. Nix and Nip3 form a subfamily of pro-apoptotic mitochondrial proteins. J Biol Chem. 1999;274(1):7-10.

14. Zhang J, Loyd MR, Randall MS, Waddell MB, Kriwacki RW, Ney PA. A short linear motif in BNIP3L (NIX) mediates mitochondrial clearance in reticulocytes. Autophagy. 2012;8(9):1325-32.

15. Novak I, Kirkin V, McEwan DG, Zhang J, Wild P, Rozenknop A, et al. Nix is a selective autophagy receptor for mitochondrial clearance. EMBO Rep. 2010; 11(1):45-51.

16. Schweers RL, Zhang J, Randall MS, Loyd MR, Li W, Dorsey FC, et al. NIX is required for programmed mitochondrial clearance during reticulocyte maturation. Proc Natl Acad Sci U S A. 2007;104(49):19500-5.

17. Sandoval H, Thiagarajan P, Dasgupta SK, Schumacher A, Prchal JT, Chen M, et al. Essential role for nix in autophagic maturation of erythroid cells. Nature. 2008;454(7201):232-5.

18. Bellot G, Garcia-Medina R, Gounon P, Chiche J, Roux D, Pouysségur J, et al. Hypoxia-induced autophagy is mediated through hypoxia-inducible factor induction of BNIP3 and BNIP3L via their BH3 domains. Mol Cell Biol. 2009; 29(10):2570-81.

19. Chen Y, Lewis W, Diwan A, Cheng EHY, Matkovich SJ, Dorn GW 2nd. Dual autonomous mitochondrial cell death pathways are activated by nix/BNip3L and induce cardiomyopathy. Proc Natl Acad Sci U S A. 2010;107(20):903542. 
20. Diwan A, Matkovich SJ, Yuan Q, Zhao W, Yatani A, Brown JH, et al. Endoplasmic reticulum-mitochondria crosstalk in NIX-mediated murine cell death. J Clin Invest. 2009;119(1):203-12.

21. Prabakaran S, Swatton JE, Ryan MM, Huffaker SJ, Huang J-J, Griffin JL, et al. Mitochondrial dysfunction in schizophrenia: evidence for compromised brain metabolism and oxidative stress. Mol Psychiatry. 2004;9(7):684-97.

22. Kung $L$, Roberts $R C$. Mitochondrial pathology in human schizophrenic striatum: a postmortem ultrastructural study. Synapse. 1999:31(1):67-75.

23. Bloom FE. Advancing a neurodevelopmental origin for schizophrenia. Arch Gen Psychiatry. 1993;50(3):224-7.

24. Rajasekaran A, Venkatasubramanian G, Berk M, Debnath M. Mitochondrial dysfunction in schizophrenia: pathways, mechanisms and implications. Neurosci Biobehav Rev. 2015:48:10-21.

25. Vos M, Lauwers E, Verstreken P. Synaptic mitochondria in synaptic transmission and organization of vesicle pools in health and disease. Front Synaptic Neurosci. 2010;2:139.

26. Hollenbeck PJ. Mitochondria and neurotransmission: evacuating the synapse. Neuron. 2005;47(3):331-3.

27. Guo L, Tian J, Du H. Mitochondrial dysfunction and synaptic transmission failure in Alzheimer's disease. J Alzheimers Dis. 2017;57(4):1071-86.

28. Wan Q-F, Nixon E, Heidelberger R. Regulation of presynaptic calcium in a mammalian synaptic terminal. J Neurophysiol. 2012;108(11):3059-67.

29. Y-g T, Zucker RS. Mitochondrial involvement in post-tetanic potentiation of synaptic transmission. Neuron. 1997;18(3):483-91.

30. Fisher SA, Fischer TM, Carew TJ. Multiple overlapping processes underlying short-term synaptic enhancement. Trends Neurosci. 1997;20(4):170-7.

31. Genome Analysis Toolkit. https://software.broadinstitute.org/gatk. Accessed 13 Aug 2019

32. Li H, Durbin R. Fast and accurate long-read alignment with burrowswheeler transform. Bioinformatics. 2010;26(5):589-95.

33. Wang K, Li M, Hakonarson H. ANNOVAR: functional annotation of genetic variants from high-throughput sequencing data. Nucleic Acids Res. 2010; 38(16):e164.

34. Clustal Omega. http://www.ebi.ac.uk/Tools/msa/clustalo/. Accessed 25 Aug 2019.

35. Shen J, Li Z, Chen J, Song Z, Zhou Z, Shi Y. SHEsisPlus, a toolset for genetic studies on polyploid species. Sci Rep. 2016;6:24095.

36. Li Z, Zhang Z, He Z, Tang W, Li T, Zeng Z, et al. A partition-ligationcombination-subdivision em algorithm for haplotype inference with multiallelic markers: update of the SHEsis (http://analysis.bio-x.cn). Cell Res. 2009;19(4):519-23.

37. Shi YY, He L. SHEsis, a powerful software platform for analyses of linkage disequilibrium, haplotype construction, and genetic association at polymorphism loci. Cell Res. 2005;15(2):97-8.

38. Higgins JPT, Thompson SG, Deeks JJ, Altman DG. Measuring inconsistency in meta-analyses. BMJ (Clinical research ed). 2003;327(7414):557-60.

39. 1000genomes .http://phase3browser.1000genomes.org/Homo_sapiens/Info/ Index. Accessed 20 Nov 2019.

40. ExAC Browser (Beta) | Exome Aggregation Consortium. http://exac. broadinstitute.org. Accessed 13 Aug 2019.

41. NHLBI. Exome sequencing project (ESP). Exome Variant Server. http://evs.gs. washington.edu/EVS. .

42. PolyPhen-2. http://genetics.bwh.harvard.edu/pph2/. Accessed 13 Aug 2019.

43. MutationTaster . http://www.mutationtaster.org. Accessed 13 Aug 2019.

44. SIFT .http://sift.jcvi.org/. Accessed 13 Aug 2019.

45. Chun S, Fay JC. Identification of deleterious mutations within three human genomes. (1549-5469 (Electronic)).

46. CADD https://cadd.gs.washington.edu/snv. Accessed 20 March 2020

47. Wu Y, Yao Y-G, Luo X-J. SZDB: a database for schizophrenia genetic research. Schizophr Bull. 2017;43(2):459-71.

48. SZDB2.0 .http://www.szdb.org/. Accessed 21 Nov 2019

49. Mayr C. Regulation by 3'-untranslated regions. Annu Rev Genet. 2017;51: 171-94.

50. Andreassi C, Riccio A. To localize or not to localize: mRNA fate is in $3^{\prime} U T R$ ends. Trends Cell Biol. 2009;19(9):465-74.

51. Yang J, Guo X, Zhu L, Huang J, Long J, Chen Q, et al. Rs7219 regulates the expression of GRB2 by affecting miR-1288-mediated inhibition and contributes to the risk of schizophrenia in the Chinese Han population. Cell Mol Neurobiol. 2019;39(1):137-47.

52. Shi S, Leites C, He D, Schwartz D, Moy W, Shi J, et al. MicroRNA-9 and microRNA-326 regulate human dopamine D2 receptor expression, and the
microRNA-mediated expression regulation is altered by a genetic variant. J Biol Chem. 2014;289(19):13434-44.

53. Wu S, Zhang R, Nie F, Wang X, Jiang C, Liu M, et al. MicroRNA-137 inhibits EFNB2 expression affected by a genetic variant and is expressed aberrantly in peripheral blood of schizophrenia patients. EBioMedicine. 2016;12:133-42.

54. Kim AH, Reimers M, Maher B, Williamson V, McMichael O, McClay JL, et al. MicroRNA expression profiling in the prefrontal cortex of individuals affected with schizophrenia and bipolar disorders. Schizophr Res. 2010; 124(1-3):183-91.

55. SNPinfo Web Server. https://snpinfo.niehs.nih.gov/. Accessed 5 Sept. 2019.

56. Sarachana T, Zhou R, Chen G, Manji HK, Hu WW. Investigation of posttranscriptional gene regulatory networks associated with autism spectrum disorders by microRNA expression profiling of lymphoblastoid cell lines. Genome Med. 2010;2(4):23.

57. Hunsberger JG, Fessler EB, Chibane FL, Leng Y, Maric D, Elkahloun AG, et al. Mood stabilizer-regulated miRNAs in neuropsychiatric and neurodegenerative diseases: identifying associations and functions. Am J Transl Res. 2013;5(4):450-64.

58. Bastle RM, Oliver RJ, Gardiner AS, Pentkowski NS, Bolognani F, Allan AM, et al. In silico identification and in vivo validation of miR-495 as a novel regulator of motivation for cocaine that targets multiple addiction-related networks in the nucleus accumbens. Mol Psychiatry. 2018;23(2):434-43.

59. Wang J, Le T, Wei R, Jiao Y. Knockdown of JMJD1C, a target gene of hsamiR-590-3p, inhibits mitochondrial dysfunction and oxidative stress in MPP+-treated MES23.5 and SH-SY5Y cells. Cell Mol Biol (Noisy-le-grand). 2016:62(3):39-345

60. Yuan Y, Zheng Y, Zhang X, Chen Y, Wu X, Wu J, et al. BNIP3L/NIX-mediated mitophagy protects against ischemic brain injury independent of PARK2. Autophagy. 2017;13(10):1754-66.

61. Ma J, Ni H, Rui Q, Liu H, Jiang F, Gao R, et al. Potential roles of NIX/BNIP3L pathway in rat traumatic brain injury. 2019. 28(5):585-95.

\section{Publisher's Note}

Springer Nature remains neutral with regard to jurisdictional claims in published maps and institutional affiliations.

\section{Ready to submit your research? Choose BMC and benefit from:}

- fast, convenient online submission

- thorough peer review by experienced researchers in your field

- rapid publication on acceptance

- support for research data, including large and complex data types

- gold Open Access which fosters wider collaboration and increased citations

- maximum visibility for your research: over $100 \mathrm{M}$ website views per year

At BMC, research is always in progress.

Learn more biomedcentral.com/submissions 